\title{
Chapter 149 \\ Production of Hypernuclei and Strange Particles in Spallation Reactions at a Few GeV Using an Intranuclear Cascade Approach
}

\author{
Jean-Christophe David, Jason Hirtz, Jose Luis Rodríguez-Sánchez, \\ Alain Boudard, Joseph Cugnon, Sylvie Leray, Ingo Leya, Davide Mancusi \\ and Georg Schnabel
}

\begin{abstract}
Motivated by a renewed interest in studies of hypernuclei, the strangeness degree of freedom was implemented in the intranuclear cascade model INCL. This model takes care of the first stage of reactions between a nucleon (or a light cluster) and a nucleus at energies from a few tens of $\mathrm{MeV}$ up to a few $\mathrm{GeV}$. After emission of fast particles, a hot remnant nucleus is produced and another model, combined to INCL, handles the de-excitation (Abla in our case). The main ingredients are discussed and we compare the results to experimental data.
\end{abstract}

\footnotetext{
J.-C. David (凶) · J. Hirtz · J. L. Rodríguez-Sánchez · A. Boudard · S. Leray · G. Schnabel IRFU, CEA, Université Paris-Saclay, 91191 Gif-sur-Yvette, France

e-mail: jcdavidb@free.fr; jean-christophe.david@cea.fr

J. Hirtz · I. Leya

Space Research and Planetary Sciences, Physics Institute, University of Bern, Sidlerstrasse 5, 3012 Bern, Switzerland

\section{J. L. Rodríguez-Sánchez}

Universidad de Santiago de Compostela, 15782 Santiago de Compostela, Spain

GSI-Helmholtzzentrum für Schwerionenforschung $\mathrm{GmbH}$,

64291 Darmstadt, Germany

J. Cugnon

AGO department, University of Liège, allée du 6 août 19, bâtiment B5,

4000 Liège, Belgium

D. Mancusi

Den-Service d'étude des réacteurs et de mathématiques appliquées (SERMA),

CEA, Université Paris-Saclay, 91191 Gif-sur-Yvette, France 


\subsection{Introduction}

The hypernucleus is a suitable laboratory to study YN and YY interactions and new experiments, either in progress or planned, in several facilities (JPARC, MAMI, JLab, GSI, FAIR, ...), makes the topic more and more attractive. While light hypernuclei have been studied extensively, the heaviest ones are also interesting objects, for example to study the behavior of hyperons in nuclear matter (role of the $\Lambda$ in neutron stars). Our nuclear reaction code, called INCL (Liège IntraNuclear Cascade), treats reactions between light particles and nuclei with incident energies from $\sim 100 \mathrm{MeV}$ up to $\sim 15 \mathrm{GeV}$ [1]. The renewed interest in hypernucleus studies and the sound bases of the model implemented in INCL were the motivations to add K's, $\Lambda$ and $\Sigma$ 's as participant particles in INCL. The de-excitation code Abla was also upgraded. Below we give first the ingredients required to perform the calculations and, second, we compare the obtained results to experimental data and to predictions from other model codes.

\subsection{Ingredients}

An intranuclear cascade is a series of collisions between hadrons. The main ingredients to simulate such collisions are the elementary cross sections, the particle momenta and charges in the output channels and the nuclear potential felt by all particles. It must be stressed that in INCL the resonances are not considered as participant particles (except the $\Delta(1232)$ ); Only their decay products play a role.

Adding the four Kaons $\left(K^{+}, K^{-}, K^{0}, \overline{\mathrm{K}}^{0}\right)$, the $\Lambda$ and the three $\Sigma\left(\Sigma^{-}, \Sigma^{0}, \Sigma^{+}\right)$, implies more than 400 new channels, when isospin is considered. All details are described in a recent published paper [2]. Here we only draw the attention to the difficulties to obtain all necessary information. As an example, if one excepts the $\Delta$-induced and inclusive reactions, 382 isospin channels are involved and therefore 382 cross section parametrizations. Only $17 \%$ of them can be obtained by using experimental data. Considering isospin symmetry at the initial and final states of some binary collisions, an extra $18 \%$ is obtained from relations between known and unknown cross sections. Still relying on isospin symmetry, but, this time, at each vertex of tree Feynman diagrams used within a hadron exchange model, 37\% of the cross sections is determined by ratios between known and unknown cross sections. The remaining cross sections are based on models or hypotheses.

Once a reaction between two hadrons in the nucleus is chosen, charges and momenta of the particles in the final state must be defined. Concerning the charges, Clebsch Gordan coefficients are used as far as possible if two or more particles are involved. When the number of particles increases, additional models are sometimes needed to remove ambiguities. Otherwise, as in the case of the inclusive reactions, results of other codes are used to determine the number and type of particles emitted. Momenta are taken from measurements or by assuming isotropy or considering a phase-space distribution. Here again, the reliability of our approach may differ from channel to channel. 
For the potential, the values used in this study are: $\mathrm{V}_{\Lambda}=-28 \mathrm{MeV}, \mathrm{V}_{\Sigma}=16 \mathrm{MeV}$, $\mathrm{V}_{K^{+}}=25 \mathrm{MeV}, \mathrm{V}_{K^{-}}=-60 \mathrm{MeV}, \mathrm{V}_{K^{0}}=25 \mathrm{MeV}, \mathrm{V}_{\bar{K}^{0}}=-50 \mathrm{MeV}$. The latest value implemented in INCL for $\mathrm{V}_{\Lambda}$ is actually mass dependent and will be discussed. However, the results presented here were performed with the value of $-28 \mathrm{MeV}$, which is very close to the new $\mathrm{V}_{\Lambda}(\mathrm{A})$ for the light and medium mass nuclei.

\subsection{Results}

Comparisons to experimental data and other models are the only way to test the reliability of a model and to try to understand remaining deficiencies. Most of the measured data in this domain are related to the $\mathrm{K}^{+}$production. However, some other data exist and were used to benchmark this first version of INCL considering strangeness.

\subsubsection{Particle Production}

The production of $K^{+}$is well described by INCL. This is illustrated with Fig. 149.1, where we can see also the important role played by the $\Delta$-induced reactions, especially at low energies. While at highest energies the parametrization used for those

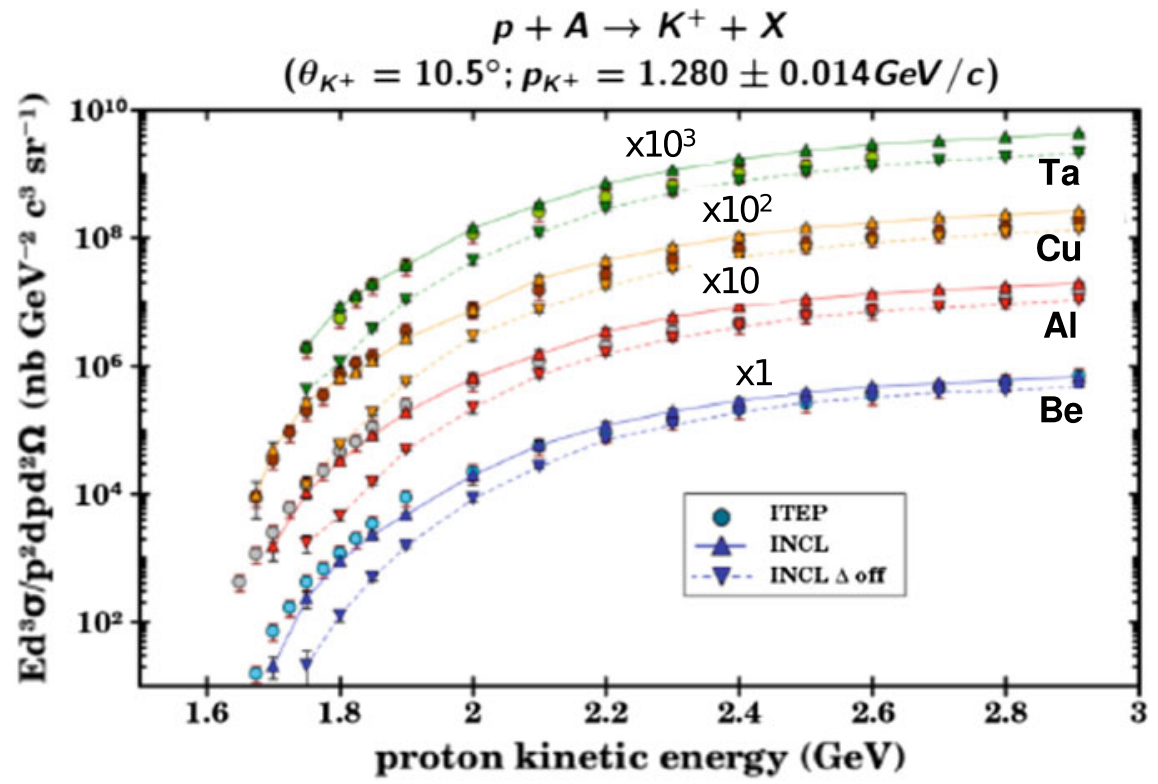

Fig. 149.1 Invariant production cross sections of $\mathrm{K}^{+}$emitted with a momentum of $1.28 \pm 0.014$ $\mathrm{GeV} / \mathrm{c}$ at an angle of $10.5^{\circ}$ for four targets as a function of proton projectile energy. Experimental data are from [4] (circles) and are compared to INCL with (up-oriented triangles) and without (down-oriented triangles) $\Delta$-induced Kaon production 
reactions [3] could be improved, benchmarks (not shown here) above $10 \mathrm{GeV}$ still exhibit very good behaviors of INCL. In the forward direction more experimental data are needed to really know the situation. The conclusions for the $K^{-}$are different. The spectra are well reproduced except for low momenta. This seems to indicate that in INCL some production channels are missing, especially $Y N \rightarrow \overline{\mathrm{K}} N N$. Regarding the $\Lambda$, we compared the INCL results to the HADES rapidity spectrum for the reaction $\mathrm{p}(3.5 \mathrm{GeV})+\mathrm{Nb}$. INCL matches rather well the HADES data [5], except for rapidi-
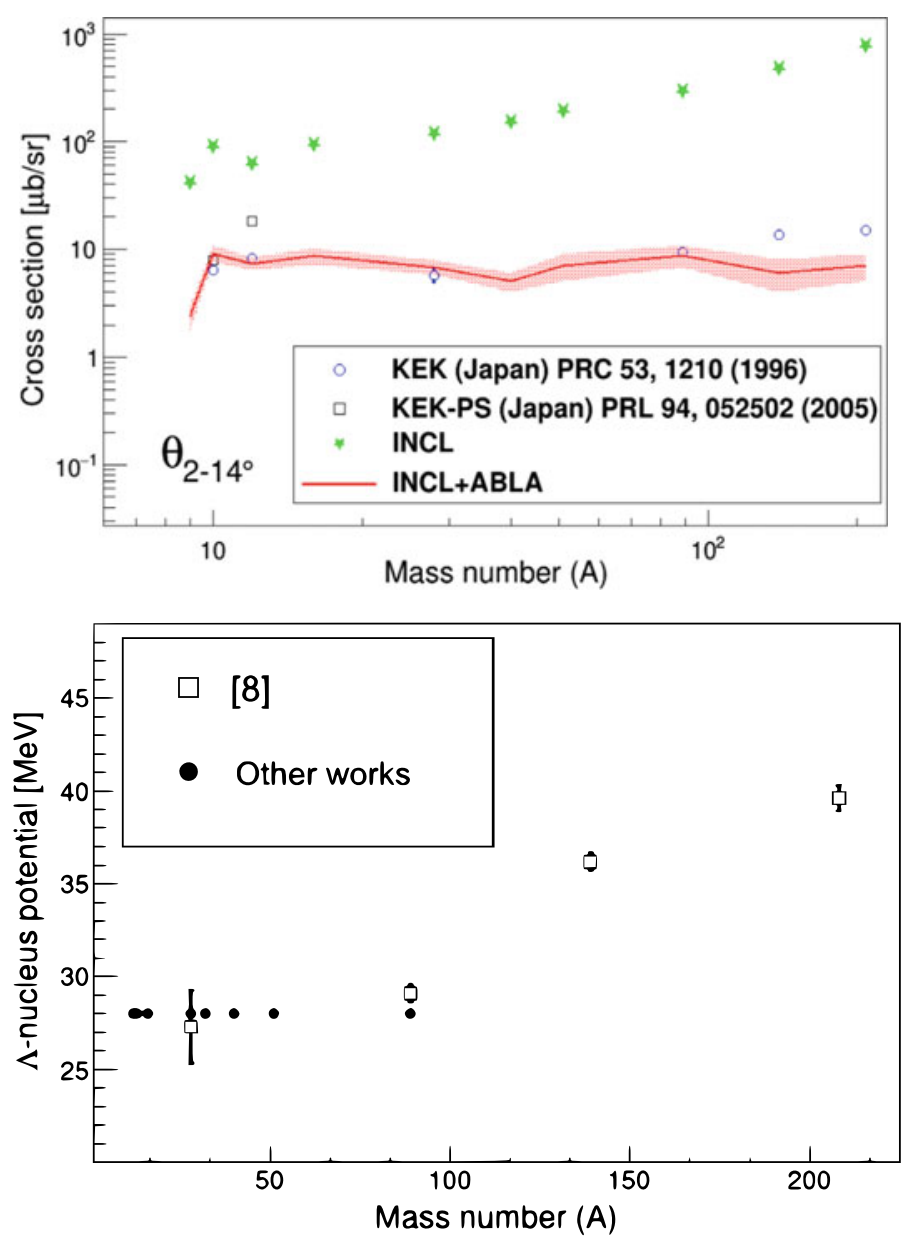

Fig. 149.2 Hypernucleus production cross section in function of the mass $\operatorname{target}$ for ${ }^{A} \mathrm{X}\left(\pi^{+}, K^{+}\right){ }_{\Lambda}^{A} \mathrm{X}$ reactions, with incident energies of 1.06 and $1.048 \mathrm{GeV} / \mathrm{c}$ (upper part). Experimental data are from [6] (circles) and [7] (squares). Are plotted INCL hyperremnant production (green stars) and INCL-Abla result (solid red line surrounded by a red band for the uncertainties). Is plotted also $\Lambda$-Nucleus potential fitted with the above experimental data in function of the mass target (lower part) 
ties larger than 0.8 . This discrepancy, possibly due to the low transverse momenta, must be now carefully studied to understand the differences between experimental data and model predictions.

\subsubsection{Hypernucleus Production}

Fig. 149.2 shows the production of hypernuclei as a function of the mass target for cases in which one, and only one, $K^{+}$is emitted by $\pi^{+}$-induced reactions $\left({ }^{A} \mathrm{X}\left(\pi^{+}, K^{+}\right){ }_{\Lambda}^{A} \mathrm{X}\right)$. INCL fits very well the KEK experimental data, except an underestimate of the heaviest nuclei. This deviation has been used to redefine the potential, assuming a dependence on the mass of the nucleus. The thus updated INCL model produces a perfect fit of the measurements (not shown here). This increase of the potential with the mass, i.e., with the nucleon-isospin asymmetry, could indicate a role of the $\Lambda \mathrm{nn}$ interaction. All details are in [8].

\subsection{Conclusion}

The strange particles $K^{+}, K^{-}, K^{0}, \overline{\mathrm{K}}^{0}, \Lambda, \Sigma^{-}, \Sigma^{0}$ and $\Sigma^{+}$are implemented into the intranuclear cascade code INCL. Since the de-excitation code Abla is usually combined to INCL for a full simulation of spallation reactions, Abla has been also upgraded by adding $\Lambda$ evaporation and hyperfission. The results obtained by the codes, and especially INCL, are very encouraging. The main mechanisms are incorporated and now it is time for improvements. The $\Delta$-induced strangeness production is probably overestimated when energy goes up and some strangeness exchange reactions must be added, like $\Lambda N \rightarrow \overline{\mathrm{K}} N N$, to better reproduce $K^{-}$production. Other aspects must also be studied, like the momentum distribution of the emitted particles. However, the lack of experimental data to get better elementary ingredients and to benchmark carefully our model makes the task difficult.

\section{References}

1. Mancusi, D., et al.: Eur. Phys. J. A 53, 80 (2017)

2. Hirtz, J., et al.: Eur. Phys. J. Plus 133, 436 (2018)

3. Tsushima, K., et al.: Phys. Rev. C 59, 369 (1999)

4. Akindinov, A.V., et al.: J. Exp. Theo. Phys. Lett. 72, 100 (2000)

5. Agakishiev, G.: HADES collaboration. Eur. Phys. J. A 50, 81 (2014)

6. Hasegawa, T., et al.: Phys. Rev. C 53, 1210 (1996)

7. Saha, P.K., et al.: Phys. Rev. Lett. 94, $052502(2005)$

8. Rodríguez-Sánchez, J.L., et al.: Phys. Rev. C 98, 021602(R) (2018) 